\title{
A single-blind study of podophyllotoxin cream $0.5 \%$ and podophyllotoxin solution $0.5 \%$ in male patients with genital warts
}

\author{
Carsten Sand Petersen, Tove Agner, Vibeke Ottevanger, John Larsen, Lisbeth Ravnborg
}

Department of

Dermato-venereology,

Gentofte Hospital,

University of

Copenhagen, Denmark

C S Petersen

Department of

Dermato-venereology, Bispebjerg Hospital, University of

Copenhagen, Denmark

T Agner

V Ottevanger

Department of

Dermato-venereology,

Rigshospitalet,

University of

Copenhagen, Denmark

J Larsen

L Ravnborg

Correspondence to:

Carsten Sand Petersen

M.D., Department of

Dermato-venereology

Bispebjerg Hospital

DK-2400 Copenhagen,

Denmark.

Accepted for publication

6 September 1995

\begin{abstract}
Objective-To evaluate the efficacy-safety ratio of a new topical podophyllotoxin cream $0.5 \%$ compared with podophyllotoxin solution $0.5 \%$ (Condyline) in male patients with genital warts.

Methods-In an observer-blinded controlled study a total of 136 and 133 wart lesions were treated with podophyllotoxin cream $0.5 \%$ and podophyllotoxin solution $0.5 \%$, respectively. The preparations were applied twice daily for 3 days, repeated with 4 days intervals for a minimum of two and a maximum of four treatment cycles.

Results-At the conclusion of the study ( 8 weeks after completion of therapy) a significant reduction in mean wart area was observed in both the cream group $(87 \cdot 7$, SD 8.4 to $20 \cdot 6$, SD $2 \cdot 7$ ) and in the solution group $(92 \cdot 3$, SD $7 \cdot 5$ to $21 \cdot 5$, SD $2 \cdot 8)$ $(p<0.01)$. At the same time all treated warts had completely cleared in $63 \%$ of patients in both study groups. Mild to moderate side effects occurred to the same extent in both podophyllotoxin cream and podophyllotoxin solution recipients.

Conclusion-We conclude that podophyllotoxin $0.5 \%$ administered in a cream formulation does not give additional clinical benefits when compared with podophyllotoxin solution $0.5 \%$ in male patients with external genital warts.
\end{abstract}

(Genitourin Med 1995;71:391-392)

Keywords: Genital warts; podophyllotoxin

\section{Introduction}

Purified ethanolic solutions of $0.5 \%$ podophyllotoxin have in recent years been available for self-treatment of external genital warts in both male $^{1-3}$ and female patients. ${ }^{45}$ The $0.5 \%$ cream formulation of podophyllotoxin was developed in order to make application more convenient with an efficacy-safety profile comparable to that of podophyllotoxin solution $0.5 \%$. The use of a solution and applicator is convenient in the self-treatment of warts that are visible to the patient. When warts are inaccessible, or patients find it difficult or inconvenient to use a solution and applicator, then an alternative pharmaceutical preparation such as a cream could be preferred.

This study was designed to evaluate the comparative efficacy and safety of patient- applied podophyllotoxin cream $0.5 \%$ versus podophyllotoxin solution $0.5 \%$ in the treatment of external genital warts in male patients.

\section{Patients and methods}

The study was conducted and approved by the appropriate institutional review boards in three dermato-venereological centres in Copenhagen, Bispebjerg Hospital, Rigshospitalet and Gentofte Hospital which enrolled 14, 12 and 10 patients, respectively. After informed consent was obtained the patients were randomly and observer-blind assigned to one of two study groups. One group of patients received podophyllotoxin in an alcoholic solution $0.5 \%$ (Condyline) twice daily for three days repeated with four days interval for a minimum of two and a maximum of four treatment cycles. Patients in the other group were treated with a cream formulation containing podophyllotoxin $0.5 \%$ applied as described above. The cream was administered by fingertip application.

Patients who had been given a diagnosis of condyloma acuminatum were enrolled if they were in general good health and if they had not received topical or systemic antiviral or antiwart therapy within the last four weeks.

Clinical efficacy was determined by quantitative assessment of the treated warts. The two larger perpendicular dimensions of the warts were measured and the wart area was defined as the product of those two measurements. Clearing of warts were defined as the disappearance of all treated warts in a patient.

Safety was assessed by questioning and examining patients for local reactions indicating intolerance of the treatment. Adverse reactions including tenderness, burning, pain, erythema, erosions and oedema were noticed and if present described as mild, moderate or severe.

The distributions of the demographic and efficacy and safety data on the two treatment groups were compared with the use of analyses of variance, the Wilcoxon test, Fisher's exact test and the chi square test.

\section{Results}

Clinical efficacy

The demographic data of included patients are shown in table 1 . The majority of treated wart lesions were penile and the mean wart area was comparable in the two treatment groups. Application of podophyllotoxin solution $0.5 \%$ was associated with a rapid and significant 
Table 1 Demographic data on patients

\begin{tabular}{lcc}
\hline & \multicolumn{2}{l}{ Study group } \\
\cline { 2 - 3 } Characteristic & $\begin{array}{l}\text { Podophyllotoxin } \\
\text { solution 0.5\% }\end{array}$ & $\begin{array}{l}\text { Podophyllotoxin } \\
\text { cream 0.5\% }\end{array}$ \\
\hline Mean age (year) & 24 & 22 \\
Previous therapy (\%) & 30 & 28 \\
Duration of warts (months) & $2 \cdot 5$ & $3 \cdot 5$ \\
Medium number of warts per patient & 7 & 8 \\
Mean (SD) wart area (mm ${ }^{2}$ ) & $92 \cdot 3(7 \cdot 5)$ & $87 \cdot 7(8 \cdot 4)$ \\
Location of warts (no) & 122 & 126 \\
Penile & 5 & 7 \\
Perianal & 4 & 3 \\
Urethral & & \\
\hline
\end{tabular}

Table 2 Reduction in wart area during treatment of condyloma acuminatum with podophyllotoxin cream $0.5 \%$ and podophyllotoxin solution $0.5 \%$ in male patients. $P$ values $(*, \dagger)$ indicates significant reduction from baseline. No significant difference was found between treatment groups

\begin{tabular}{llllll}
\hline & \multicolumn{2}{l}{$\begin{array}{l}\text { Podophyllotoxin } \\
\text { cream 0.5\% }\end{array}$} & & $\begin{array}{l}\text { Podophyllotoxin } \\
\text { solution 0.5\% }\end{array}$ \\
\cline { 2 - 3 } \cline { 5 - 6 } $\begin{array}{l}\text { Week of } \\
\text { study }\end{array}$ & $\begin{array}{l}\text { Total no } \\
\text { of lesions }\end{array}$ & $\begin{array}{l}\text { Mean }(S D) \\
\text { wart area }\end{array}$ & & $\begin{array}{l}\text { Total no } \\
\text { of lesions }\end{array}$ & $\begin{array}{l}\text { Mean (SD) } \\
\text { wart area }\end{array}$ \\
\hline 0 & 136 & $87 \cdot 7(8 \cdot 4)$ & 133 & $92 \cdot 3(7 \cdot 5)$ \\
1 & 122 & $76 \cdot 0(5 \cdot 6)$ & 85 & $59 \cdot 7(6 \cdot 7)^{\star}$ \\
2 & 102 & $66 \cdot 4(8 \cdot 7)$ & 72 & $47 \cdot 0(4 \cdot 8)^{\star}$ \\
3 & 80 & $52 \cdot 2(7 \cdot 2)^{\star}$ & & 38 & $25 \cdot 2(3 \cdot 2) \dagger$ \\
5 & 51 & $33 \cdot 6(4 \cdot 2)^{\star}$ & & 12 & $9 \cdot 2(0 \cdot 8) \dagger$ \\
6 & 44 & $29 \cdot 2(3 \cdot 2) \dagger$ & 5 & $4 \cdot 5(0 \cdot 5) \dagger$ \\
12 & 32 & $20 \cdot 6(2 \cdot 7) \dagger$ & 29 & $21 \cdot 5(2 \cdot 8) \dagger$ \\
\hline
\end{tabular}

${ }^{\star} \mathrm{p}<0.05 ; \mathrm{tp}<0.01$.
Evaluation of safety

Local adverse reactions graded mild to moderate were noticed in $35 \%$ of podophyllotoxin solution recipients compared with $40 \%$ of podophyllotoxin cream recipients (none significant). Separate analyses of tenderness, burning, pain, erythema, erosions and oedema did not show differences between the two treatment groups. Only one patient treated with podophyllotoxin cream $0.5 \%$ did not complete the trial for reasons not associated with therapy.

\section{Discussion}

The final cure rate obtained with the two podophyllotoxin preparations is well within the range seen in previous published studies. ${ }^{1-5}$ In this trial an advantage of podophyllotoxin cream $0.5 \%$ over a well-known ethanolic solution of podophyllotoxin $0.5 \%$ could not be documented. Neither the clinical efficacy nor safety aspects were in favour of podophyllotoxin cream $0.5 \%$. It is possible that a cream formulation of podophyllotoxin may be easier to apply and therefore improve the compliance in female patients with external genital warts. A drawback of a cream formulation, however, may be a greater rate of adverse reactions, due to spreading of the cream over a larger area, as reported in a recently published study in female patients. ${ }^{6}$ lesions, whereas the effect of podophyllotoxin $0.5 \%$ cream was somewhat protracted (table 2). A total of $95 \%$ of patients treated with podophyllotoxin $0.5 \%$ solution were free of warts two weeks after completion of therapy, but only $63 \%$ remained clear at the final control-visit (week 12). In comparison $63 \%$ of patients treated with podophyllotoxin $0.5 \%$ cream were wart free two weeks after stopping therapy. However, the final cure rate of $63 \%$ in cream recipients was identical to that obtained in solution recipients. No difference could be detected in the overall efficacy of the two formulations of podophyllotoxin (table 2). The number of applications of either podophyllotoxin cream or solution was not significantly different in the two treatment groups.
1 Krogh Von G. Penile condylomata acuminatum: An experimental model for evaluation of topical self-treatment with $0.5-1.0 \%$ ethanolic preparations of podophyllotoxin for three days. Sex Trans Dis 1981;8:179-86. $0.5 \% v$ podophyllotoxin $20 \%$ to treat penile warts. Genitourin Med 1986;64:263-5. Patient-applied podofilox for treatment of genital warts. Lancet 1989;8642:831-3.

4 Baker DA, Douglas JM, Buntin DM, et al. Topical podofilox in the treatment of condylomata acuminata in women. Obstet Gynecol 1990;76:656-9.

5 Greenberg MO, Rutledge LH, Reid R, et al. A doubleblind, randomized trial of $0.5 \%$ podofilox and placebo for the rreatment of genital warts in women. Obstet Gynecol 1991;77:735-9.

6 Mazurkiewicz W, Jablonska S. Clinical efficacy of Condyline $(0.5 \%$ podophyllotoxin) solution and cream versus podophyllin in the treatment of external condyloma acuminata. $\mathcal{F}$ Dermatol Treat 1990;1:123-5.
2 Edwards A, Atma-Ram A, Thin RN. Podophyllotoxin

3 Beutner KR, Conant MA, Friedman-Kien AE, et al. 